\title{
A Short Note on Compressed Sensing with Partially Known Signal Support
}

\author{
Technical Report: TR-LJ-2009.02 (Updated Version) \\ L. Jacques* \\ Communications and Remote Sensing Laboratory (TELE) \\ Université catholique de Louvain (UCL) \\ Louvain-la-Neuve, Belgium
}

October 24, 2018

\begin{abstract}
This short note studies a variation of the Compressed Sensing paradigm introduced recently by Vaswani et al., i.e. the recovery of sparse signals from a certain number of linear measurements when the signal support is partially known. The reconstruction method is based on a convex minimization program coined innovative Basis Pursuit DeNoise (or $i$ BPDN). Under the common $\ell_{2}$-fidelity constraint made on the available measurements, this optimization promotes the $\left(\ell_{1}\right)$ sparsity of the candidate signal over the complement of this known part.

In particular, this paper extends the results of Vaswani et al. to the cases of compressible signals and noisy measurements. Our proof relies on a small adaption of the results of Candes in 2008 for characterizing the stability of the Basis Pursuit DeNoise (BPDN) program.

We emphasize also an interesting link between our method and the recent work of Davenport et al. on the $\delta$-stable embeddings and the cancel-then-recover strategy applied to our problem. For both approaches, reconstructions are indeed stabilized when the sensing matrix respects the Restricted Isometry Property for the same sparsity order.

We conclude by sketching an easy numerical method relying on monotone operator splitting and proximal methods that iteratively solves $i$ BPDN.
\end{abstract}

Keywords: Sparse Signal Recovery, Compressed Sensing, Convex Optimization, Instance Optimality.

\section{Introduction}

The theory of Compressed Sensing (CS) [2, 10] aims at reconstructing sparse or compressible signals from a small number of linear measurements compared to the dimensionality of the signal space. In short, the signal reconstruction is possible if the underlying sensing matrix is well behaved, i.e. if it respects a Restricted Isometry Property (RIP) saying roughly that any small subset of its columns is "close" to an orthogonal basis. The signal recovery is then obtained using non-linear techniques

*Iaurent.jacques@uclouvain. be. Research supported by Belgian National Science Foundation (F.R.S.-FNRS). 
based on convex optimization promoting signal sparsity, as the Basis Pursuit DeNoise (BPDN) program [10, 5]. What makes CS more than merely an interesting theoretical concept is that some classes of randomly generated matrices (e.g. Gaussian, Bernoulli, partial Fourier ensemble, etc) satisfy the RIP with overwhelming probability. This happens as soon as their number of rows, i.e. the number of CS measurements, is higher than a few multiples of the assumed signal sparsity.

In this paper we are interested in a variation of the CS paradigm. We assume indeed that the support of the signal to recover is partially known, possibly with a certain error. As explained in [16, 17, this context is indeed well suited to the recovery of (time) sequences of sparse signals when their supports evolves slowly over time. In that case, the support of the recovered signal in a previous (discretized) time can be used to improve the reconstruction of the signal at the next time instance, either by decreasing the required number of measurements for a given quality, or by improving the reconstruction quality for a fixed number of measurements. Recovering a signal with partially known support is also of interest for certain kind of 1-D signals or images. For instance, photographic images, i.e. with positive intensities, have often many non-zero approximation coefficients in their wavelet decomposition [12]; a prior knowledge that can be favorably used in their reconstruction from CS measurements.

By adapting the proof of [1], we show in this short note that the recovery algorithm minimizing the $\ell_{1}$-norm of the signal candidate over the complement of the known support part, i.e. what we coin innovative Basis Pursuit DeNoising ( $i \mathrm{BPDN})$, has a similar stability behavior than the common Basis Pursuit DeNoise program. In particular, this extends the result of [16, 17] to the cases of noisy measurements and of compressible signals, i.e. with non-zero but fast decaying coefficients in a given sparsity basis. We show also that our method shares somehow the conclusion of the cancelthen-recover strategy designed in [9] where Authors propose a recovery algorithm that applies an orthogonal projection to separate the measurements into two components, and then recovers the known support part of the signal separately from the unknown support component.

\section{Framework and Notations}

Let $x=\Psi \alpha \in \mathbb{R}^{n}$ be a sparse or a compressible discrete signal in the sparsity basis $\Psi \in \mathbb{R}^{n \times n}$ of $\mathbb{R}^{n}$, i.e. the vector $\alpha \in \mathbb{R}^{n}$ has few non-zero or fast decaying components respectively. For the sake of simplicity, we work hereafter with the canonical basis, i.e. $\Psi=\mathrm{Id}$, identifying $\alpha$ with $x$. The present work is however valid for any orthonormal $\Psi$, e.g. the DCT or the Wavelet basis, by integrating $\Psi$ in the sensing model described in Section 3 .

We now establish some important notations. We write $\mathcal{N}=\{1, \cdots, n\}$ the index set of the vector components in $\mathbb{R}^{n}$. For any vector $u \in \mathbb{R}^{n}, u_{i}$ is the $i^{\text {th }}$ component of $u$ with $i \in \mathcal{N}, u_{S}$ is the vector equal to the components of $u$ on the set $S \subset \mathcal{N}$ and to 0 elsewhere, while $u^{l}$, with uppercase index $l \in \mathbb{N}$ to avoid confusion, is the vector obtained by zeroing all but the $l$ largest components of $u$ (in amplitude). For non-trivial basis $\Psi, u^{l}$ would be the best $l$-term approximation of $u$ in the $\ell_{2}$-norm sense. The complement of any set $S \subset \mathcal{N}$ is denoted by $S^{c}=\mathcal{N} \backslash S$, and the size of $S$ by $\# S$. The $\ell_{p}$ norm (for $p \geq 1$ ) of $u \in \mathbb{R}^{n}$ is $\|u\|_{p}^{p}=\sum_{i}\left|u_{i}\right|^{p}$, while its support is written $\operatorname{supp} u \triangleq\left\{i \in \mathcal{N}: u_{i} \neq 0\right\}$. By extension, the $\ell_{0}$ "norm" 11 is defined as $\|u\|_{0}=\# \operatorname{supp} u$.

Let us speak now of the prior knowledge that we have on the signal. In addition to the

\footnotetext{
${ }^{1}$ It is not actually a true norm since for instance it is not positive homogeneous.
} 
assumption of sparsity or compressibility, we presume that the support of the signal $x$ is partially known. In the sequel, we denote the known support part by $T \subset \mathcal{N}$, while we always refer to its size by the letter $s=\# T$. Notice that in our study nothing prevents $T$ to be corrupted by some "noise", i.e. a priori $T$ is not fully included to $\operatorname{supp} x$. Moreover, the size of $(\operatorname{supp} x) \backslash T$ is not constrained, what will matter is the values of the components of $x$ on $(\operatorname{supp} x) \backslash T$, i.e. the compressibility of $x$ outside of $T$.

\section{Sensing Model}

Following the common Compressed Sensing model, our vector $x$ is acquired by a sensing matrix $\Phi \in \mathbb{R}^{m \times n}$ subject to an additional white noise $n \in \mathbb{R}^{m}$, i.e.

$$
y=\Phi x+n,
$$

where $y \in \mathbb{R}^{m}$ is the measurement vector. In this model the noise power is assumed bounded $2^{2}$ by $\epsilon,\|n\|_{2} \leq \epsilon$.

As shown after, even if a part of the signal support is known, the stability of this sensing model, i.e. our ability to recover or approximate $x$ from $y$, is also linked to the Restricted Isometry Property (RIP) of the sensing matrix [4, 2, 3].

Explicitly, the matrix $\Phi \in \mathbb{R}^{m \times n}$ satisfies the RIP of order $q \in \mathbb{N}(q \leq n)$ and radius $0 \leq \delta_{q}<1$, if

$$
\left(1-\delta_{q}\right)\|u\|_{2}^{2} \leq\|\Phi u\|_{2}^{2} \leq\left(1+\delta_{q}\right)\|u\|_{2}^{2},
$$

for all $q$-sparse vectors $u \in \mathbb{R}^{n}$, i.e. with $\|u\|_{0} \leq q$.

\section{Reconstructing on Innovation}

Intuitively, if a part $T \subset \mathcal{N}$ of the signal support is known, a possible (non-linear) reconstruction technique of $x$ would simply consist in minimizing the sparsity of a signal candidate $u \in \mathbb{R}^{n}$ over $T^{c}$, i.e. the $\ell_{0}$-norm of $u_{T^{c}}$, subject to the common $\ell_{2}$ fidelity constraint $\|\Phi u-y\|_{2} \leq \epsilon$ as prescribed by the noise power bound. As underlined many times in the community, such a procedure would result in a combinatorial (NP-hard) problem [13. Here again an $\ell_{1}$ relaxation must be used, with possibly additional requirements on the RIP-"conditioning" of $\Phi[15,3]$.

The proposed method is a simple extension of the Modified-CS scheme defined in [16, 17]. We integrate indeed the case of corrupted measurements by defining the following optimization program, coined innovative Basis Pursuit DeNoising ( $i \mathrm{BPDN})$,

$$
\underset{u}{\operatorname{argmin}}\left\|u_{T^{c}}\right\|_{1} \text { s.t. }\|y-\Phi u\|_{2} \leq \epsilon .
$$

The term "innovative" recalls that this program tries to minimize the sparsity of the signal to be reconstructed in the unknown (or innovation) set $(\operatorname{supp} x) \backslash T$ included to $T^{c}$.

\footnotetext{
${ }^{2}$ Possibly with high probability.
} 


\section{$5 \quad i \mathrm{BPDN}$ and $\ell_{2}-\ell_{1}$ Instance Optimality}

The main result of this note provides the conditions under which the solution of $i$ BPDN is close or equal to the initial signal $x$, i.e. the so-called $\ell_{2}-\ell_{1}$ instance optimality [6]. It extends in the same time the conclusion of [16, 17] to the cases of noisy measurements and compressible signals.

Theorem 1. Under the condition of the sensing model described above, writing $\# T=s$ and given $k \in \mathbb{N}$, let us assume that the matrix $\Phi$ respects the $R I P$ of order $s+2 k$ with radius $\delta_{s+2 k} \in(0,1)$, and that its radius for the smaller order $2 k$ is $\delta_{2 k} \in(0,1)$. Then, if $\delta_{2 k}^{2}+2 \delta_{s+2 k}<1$, iBPDN has the $\ell_{2}-\ell_{1}$ instance optimality meaning that its solution $x^{*}$ respects

$$
\left\|x-x^{*}\right\|_{2} \leq C_{s, k} \epsilon+D_{s, k} e_{0}(r ; k),
$$

where $r$ is the residual $r=x-x_{T}$, and $e_{0}(r ; k)=k^{-1 / 2}\left\|r-r^{k}\right\|_{1}$ is the compressibility error ${ }^{3}$ at $k$-term of $r$. The two constants $C_{s, k}$ and $D_{s, k}$, given in the proof, depend on $\Phi$ only. For instance, for small innovation, i.e. when $k \ll s$, if $\delta_{2 k}=0.02$ and if $\delta_{s+2 k}=0.2, C_{s, k}<7.32$ and $D_{s, k}<3.35$.

Proof. We basically adapt the proof of [1] to signal with partially known support.

We define the residual $r=x-x_{T}$, with $\operatorname{supp} r=(\operatorname{supp} x) \backslash T$. Let us write $x^{*}=x+h$ with $h \in \mathbb{R}^{n}$ so that the proof amounts to bound $\|h\|_{2}$. Let $T_{0}$ be the support of the $k$ largest coefficients of the residual $r=x-x_{T}$, i.e. $T_{0}=\operatorname{supp} r^{k}$ with $T_{0} \cap T=\emptyset$.

We define next the sets $T_{j}$ for $j \geq 1$ as the support of the $k$ largest coefficients of $h_{S_{j}^{c}}=h-h_{S_{j}}$ with $S_{j}=T \cup \bigcup_{l=0}^{j-1} T_{l}$. By construction, we may observe that we got the partition $\bigcup_{l \geq 0} T_{l}=$ $(\operatorname{supp} x) \backslash T$, with $\# T_{j}=k$ and $T_{j} \cap T=T_{j} \cap T_{j^{\prime}}=\emptyset$, for $j, j^{\prime} \geq 0$ and $j \neq j^{\prime}$.

Let us write $T_{\mid 0}=T \cup T_{0}$ and $T_{\mid 01}=T \cup T_{0} \cup T_{1}$, with $\# T_{\mid 0}=s+k$ and $\# T_{\mid 01}=s+2 k$. The plan of the proof is to first bound $\left\|h_{T_{\mid 01}^{c}}\right\|_{2}$ and then $\left\|h_{T_{\mid 01}}\right\|_{2}$.

Using the triangular inequality, we have $\left\|h_{T_{\mid 01}^{c}}\right\|_{2} \leq \sum_{j \geq 2}\left\|h_{T_{j}}\right\|_{2}$. For $j \geq 1,\left\|h_{T_{j}}\right\|_{1} \geq$ $k\left\|h_{T_{j+1}}\right\|_{\infty}$ by the ordering of the $T_{j}$ 's, and therefore $\left\|h_{T_{j+1}}\right\|_{2}^{2} \leq k\left\|h_{T_{j+1}}\right\|_{\infty}^{2} \leq \frac{1}{k}\left\|h_{T_{j}}\right\|_{1}^{2}$. This leads to

$$
\left\|h_{T_{\mid 01}^{c}}\right\|_{2} \leq \frac{1}{\sqrt{k}} \sum_{j \geq 1}\left\|h_{T_{j}}\right\|_{1}=\frac{1}{\sqrt{k}}\left\|h_{T_{\mid 0}^{c}}\right\|_{1} .
$$

Since $T^{c}=T_{0} \cup T_{\mid 0}^{c}$ and $\|n\|_{2}=\|y-\Phi x\|_{2} \leq \epsilon$, and because $x^{*}$ solves $i$ BPDN, we have

$$
\left\|x_{T^{c}}\right\|_{1} \geq\left\|x_{T^{c}}+h_{T^{c}}\right\|_{1}=\left\|x_{T_{0}}+h_{T_{0}}\right\|_{1}+\left\|x_{T_{\mid 0}^{c}}+h_{T_{\mid 0}^{c}}\right\|_{1} \geq\left\|x_{T_{0}}\right\|_{1}-\left\|h_{T_{0}}\right\|_{1}-\left\|x_{T_{\mid 0}^{c}}\right\|_{1}+\left\|h_{T_{\mid 0}^{c}}\right\|_{1},
$$

and therefore,

$$
\left\|h_{T_{\mid 0}^{c}}\right\|_{1} \leq\left\|x_{T^{c}}\right\|_{1}+\left\|x_{T_{\mid 0}^{c}}\right\|_{1}+\left\|h_{T_{0}}\right\|_{1}-\left\|x_{T_{0}}\right\|_{1}=2\left\|x_{T_{\mid 0}^{c}}\right\|_{1}+\left\|h_{T_{0}}\right\|_{1}=2\left\|r-r_{T_{0}}\right\|_{1}+\left\|h_{T_{0}}\right\|_{1} .
$$

Consequently, using (1) and the equivalence of the norms $\ell_{2}$ and $\ell_{1}$, we get

$$
\left\|h_{T_{\mid 01}^{c}}\right\|_{2} \leq \sum_{j \geq 2}\left\|h_{T_{j}}\right\|_{2} \leq 2 e_{0}(r ; k)+\left\|h_{T_{0}}\right\|_{2}
$$

\footnotetext{
${ }^{3}$ It could be called also scaled $\ell_{1}$-approximation error.
} 
Let us now bound $\left\|h_{T_{01}}\right\|_{2}$. Notice that $h_{T_{\mid 01}}=h-\sum_{j \geq 2} h_{T_{j}}$, so that, using Cauchy-Schwarz,

$$
\begin{aligned}
\left\|\Phi h_{T_{\mid 01}}\right\|_{2}^{2} & =\left\langle\Phi h_{T_{\mid 01}}, \Phi h_{T_{\mid 01}}\right\rangle \\
& =\left\langle\Phi h_{T_{\mid 01}}, \Phi h\right\rangle-\left\langle\Phi h_{T_{\mid 01}}, \sum_{j \geq 2} \Phi h_{T_{j}}\right\rangle \\
& \leq\left\|\Phi h_{T_{\mid 01}}\right\|_{2}\|\Phi h\|_{2}+\sum_{j \geq 2}\left|\left\langle\Phi h_{T_{\mid 01}}, \Phi h_{T_{j}}\right\rangle\right| .
\end{aligned}
$$

By hypothesis, $\Phi$ is RIP of order $q$ and radius $\delta_{q}$ with $q \in\{2 k, s+2 k\}$. It is proved in [1] as a result of the polarization identity, that, for two vectors $u$ and $v$ of disjoint supports and of sparsity $l$ and $l^{\prime}$ respectively, if $\Phi$ is RIP of order $l+l^{\prime}$, then $|\langle\Phi u, \Phi v\rangle| \leq \delta_{l+l^{\prime}}\|u\|_{2}\|v\|_{2}$. In addition, since $x^{*}$ is solution of $i \mathrm{BPDN}$ and $x$ is a feasible point of its fidelity constraint, $\|\Phi h\|_{2} \leq$ $\left\|\Phi x^{*}-y\right\|_{2}+\|y-\Phi x\|_{2} \leq 2 \epsilon$. Therefore, combining all these considerations,

$$
\begin{aligned}
& \left\|\Phi h_{T_{\mid 01}}\right\|_{2}^{2} \leq 2 \sqrt{1+\delta_{s+2 k}} \epsilon\left\|h_{T_{\mid 01}}\right\|_{2}+\sum_{j \geq 2}\left|\left\langle\Phi h_{T_{\mid 0}}+\Phi h_{T_{1}}, \Phi h_{T_{j}}\right\rangle\right| \\
& \leq 2 \sqrt{1+\delta_{s+2 k}} \epsilon\left\|h_{T_{\mid 01}}\right\|_{2}+\left(\delta_{s+2 k}\left\|h_{T_{\mid 0}}\right\|_{2}+\delta_{2 k}\left\|h_{T_{1}}\right\|_{2}\right) \sum_{j \geq 2}\left\|h_{T_{j}}\right\|_{2} \\
& \leq 2 \sqrt{1+\delta_{s+2 k}} \epsilon\left\|h_{T_{\mid 01}}\right\|_{2}+\mu_{s, k}\left\|h_{T_{\mid 01}}\right\|_{2} \sum_{j \geq 2}\left\|h_{T_{j}}\right\|_{2},
\end{aligned}
$$

with $\mu_{s, k}=\sqrt{\delta_{s+2 k}^{2}+\delta_{2 k}^{2}}$.

Since $\left(1-\delta_{s+2 k}\right)\left\|h_{T_{\mid 01}}\right\|_{2}^{2} \leq\left\|\Phi h_{T_{\mid 01}}\right\|_{2}^{2}$, simplifying the last expression and using (2) lead to

$$
\left(1-\delta_{s+2 k}\right)\left\|h_{T_{\mid 01}}\right\|_{2} \leq 2 \sqrt{1+\delta_{s+2 k}} \epsilon+\mu_{s, k}\left(2 e_{0}(r ; k)+\left\|h_{T_{0}}\right\|_{2}\right),
$$

or, since $\left\|h_{T_{0}}\right\|_{2} \leq\left\|h_{T_{\mid 01}}\right\|_{2}$,

$$
\left\|h_{T_{\mid 01}}\right\|_{2} \leq \alpha \epsilon+\beta e_{0}(r ; k),
$$

with $\alpha=2 \sqrt{1+\delta_{s+2 k}} /\left(1-\delta_{s+2 k}-\mu_{s, k}\right)$ and $\beta=2 \mu_{s, k} /\left(1-\delta_{s+2 k}-\mu_{s, k}\right)$.

Finally, using again (2),

$$
\|h\|_{2} \leq\left\|h_{T_{\mid 01}}\right\|_{2}+\left\|h_{T_{\mid 01}^{c}}\right\|_{2} \leq \alpha \epsilon+(\beta+2) e_{0}(r ; k)+\left\|h_{T_{0}}\right\|_{2} \leq C_{s, k} \epsilon+D_{s, k} e_{0}(r ; k),
$$

with

$$
C_{s, k}=\frac{4 \sqrt{1+\delta_{s+2 k}}}{1-\delta_{s+2 k}-\mu_{s, k}}
$$

and

$$
D_{s, k}=2 \frac{1+\mu_{s, k}-\delta_{s+2 k}}{1-\delta_{s+2 k}-\mu_{s, k}} .
$$

The denominator of these two constants makes sense only if $1-\delta_{s+2 k}-\mu_{s, k}>0$, i.e. if $\delta_{2 k}^{2}+$ $2 \delta_{s+2 k}<1$, which provides the announced reconstruction condition. 


\section{Observations}

Some observations may be realized from Theorem 1 First, in the case where there is no knowledge about the signal support, i.e. $T=\emptyset$ and $s=0$, we do find the previous sufficient condition of [1] characterizing when BPDN satisfies the $\ell_{2}-\ell_{1}$ instance optimality, namely $\delta_{2 k}<\sqrt{2}-1$ as involved by $\delta_{2 k}^{2}+2 \delta_{2 k}<1$.

Second, the condition $\delta_{2 k}^{2}+2 \delta_{s+2 k}<1$ is satisfied if $\delta_{s+2 k}<\sqrt{2}-1$ since we have always $\delta_{2 k}<\delta_{s+2 k}$. This seems again a simple generalization of the previous result in [1], i.e. $i \mathrm{BPDN}$ is stable if the RIP of $\Phi$ is guaranteed over the sparsity order $s+2 k$ with a radius $\delta_{s+2 k}<\sqrt{2}-1$. Intuitively, the matrix must be sufficiently "well conditionned" to estimate both the unknown values of $x$ on the known set $T$ and the $k$ other significant values of $x$ somewhere outside of $T$. This induces somehow the required $s+2 k$ RIP sparsity order, where $s$ and $2 k$ stand for the degrees of freedom of $x$ on $T$ and on $T^{c}$ respectively.

Third, if the signal $x$ is exactly sparse, there is a $k<N-s$ such that $k=\#((\operatorname{supp} x) \backslash T)$ and $e_{0}(r ; k)=0$. Without noise on the measurements, the previous theorem guarantees therefore the perfect reconstruction of the signal, i.e. $x^{*}=x$, as obtained in [16].

Finally, the compressibility of the signal $x$ is quantified by the compressibility error $e_{0}(r, k)$. In other words, the compressibility is measured from $r=x-x_{T}$ outside of the known support part $T$ of $x$. This new measure is of course the simple generalization of the previous term $e_{0}(k)=$ $k^{-1 / 2}\left\|x-x^{k}\right\|_{1}=e_{0}(x ; k)$ introduced for instance in [1].

\section{Connection to $\delta$-stable Embeddings and the Cancel-then-Recover strategy}

Theorem 1 has an interesting connection with the recent work of Davenport et al. 9] showing that several signal processing tasks, i.e. signal detection, classification, estimation and filtering, can be realized efficiently on the compressive measurements of a signal without reconstructing it. In their work, the Authors study in particular the possibility to subtract from these measurements the influence of the known part of the signal support. Let us briefly explain that work before to compare our work with this of [9].

For this explanation, we use the framework of Section 2 with the simplifying canonical basis $\Psi=\mathrm{Id}$ and the pure sensing model $y=\Phi x$. We define also the subspace $\Sigma_{T}=\left\{u \in \mathbb{R}^{n}\right.$ : $\operatorname{supp} u \subset T\}$ and the matrix $\Omega=\Phi_{T} \in \mathbb{R}^{m \times s}$, i.e. the restriction of $\Phi$ to the columns indexed in $T \subset \mathcal{N}$. Two operators can be built from $\Omega$ and its Moore-Penrose pseudoinverse $\Omega^{\dagger}=\left(\Omega^{T} \Omega\right)^{-1} \Omega^{T}$, i.e. $P_{\Omega}=\Omega \Omega^{\dagger}$ and $P_{\Omega^{\perp}}=1-\Omega \Omega^{\dagger}$, the orthogonal projectors on the range of $\Omega$ and on the nullspace of $\Omega^{T}$ respectively.

Writing $x=x_{T}+x_{T^{c}}$, we can notice that $P_{\Omega^{\perp}} \Phi x=P_{\Omega^{\perp}} \Phi x_{T^{c}}$. In short, the influence (or interference) of $x_{T}$ on $y=\Phi x$ may be canceled without reconstructing $x$. The idea of the cancelthen-recover strategy promoted in [9] is therefore to reconstruct actually $x_{T^{c}}$ from $\widetilde{y}=\widetilde{\Phi} x=\widetilde{\Phi} x_{T^{c}}$, with $\widetilde{\Phi}=P_{\Omega^{\perp}} \Phi$. This can be done for instance by solving either the Basis Pursuit program

$$
\widetilde{x}=\underset{u}{\operatorname{argmin}}\|u\|_{1} \text { s.t. } \widetilde{y}=\widetilde{\Phi} u
$$

or an equivalent greedy method as CoSaMP [14, 9]. Of course, $\widetilde{x}_{T}=0$ since this part of $\widetilde{x}$ does not 
contribute to the fidelity constraint. It is equivalent to say that the reconstruction runs over the space $P_{\mathrm{Id}_{T}^{\perp}} \mathbb{R}^{n}$, where $P_{\mathrm{Id}_{T}^{\perp}} u=u_{T^{c}}$ for any $u \in \mathbb{R}^{n}$. Therefore, the estimation error between $\widetilde{x}$ and $x$ can be bounded over $T^{c}$.

For this purpose $\widetilde{\Phi}$ must be characterized in function of $\Phi$. This can be done by considering a generalization the Restricted Isometry Property: Given $\delta \in(0,1)$ and two spaces $\mathcal{U}, \mathcal{V} \subset \mathbb{R}^{n}$, a matrix $\Phi$ realizes a $\delta$-stable embedding of $(\mathcal{U}, \mathcal{V})$ if

$$
(1-\delta)\|u-v\|_{2}^{2} \leq\|\Phi u-\Phi v\|_{2}^{2} \leq(1+\delta)\|u-v\|_{2}^{2},
$$

for all $u \in \mathcal{U}$ and $v \in \mathcal{V}$. In particular the RIP of order $q$ and radius $\delta_{q}$ is equivalent to a $\delta_{q}$-stable embedding of $\left(\Sigma_{q},\{0\}\right)$, with $\Sigma_{q}=\left\{u \in \mathbb{R}^{n}:\|u\|_{0} \leq q\right\}$ the set of $q$-sparse signals. The following result provides then the desired characterization.

Lemma 1 (Corollary 4 in [9]). Suppose that $\Phi \in \mathbb{R}^{m \times n}$ is a $\delta$-stable embedding of $\left(\Sigma_{2 k}, \Sigma_{T}\right)$. Then $\widetilde{\Phi}$ is a $\delta /(1-\delta)$-stable embedding of $\left(P_{\mathrm{Id}_{T}} \Sigma_{2 k},\{0\}\right)$.

In particular, this Lemma implies that if $\Phi$ is RIP of order $s+2 k$ with radius $\delta_{s+2 k}$, it is then a $\delta_{s+2 k}$-stable embedding of $\left(\Sigma_{2 k}, \Sigma_{T}\right)$, and therefore, $\widetilde{\Phi}$ is RIP of order $2 k$ and radius $\delta_{s+2 k} /\left(1-\delta_{s+2 k}\right)$ over the space $P_{\operatorname{Id}_{\frac{1}{T}}} \mathbb{R}^{n} \simeq \mathbb{R}^{n-s}$. The $\ell_{2}-\ell_{1}$ instance optimality of the BP program [1] above holds if $\delta^{\prime}=\delta_{s+2 k} /\left(1-\delta_{s+2 k}\right)<\sqrt{2}-1$, i.e. if $\delta_{s+2 k}<(\sqrt{2}-1) / \sqrt{2}$. In that case,

$$
\left\|x_{T^{c}}-\widetilde{x}_{T^{c}}\right\|_{2} \leq \widetilde{D}_{\delta^{\prime}} e_{0}\left(x_{T^{c}}, k\right)=\widetilde{D}_{\delta^{\prime}} e_{0}(r, k),
$$

with $\widetilde{D}_{\delta^{\prime}}=2 \frac{1+(\sqrt{2}-1) \delta^{\prime}}{1-(\sqrt{2}+1) \delta^{\prime}}=2 \frac{1+(\sqrt{2}-2) \delta_{s+2 k}}{1-(\sqrt{2}+2) \delta_{s+2 k}}$.

In this paper, we show that $i \mathrm{BPDN}$ is optimal when $\delta_{2 k}^{2}+2 \delta_{s+2 k}<1$. This condition is weaker than the one proposed in [9], i.e. $\delta_{s+2 k}<(\sqrt{2}-1) / \sqrt{2}$, however it is interesting to notice that both consider also the RIP of order $2 s+k$ and both are stable for compressible signals. Moreover, $i$ BPDN gives guarantees for the estimation of the whole signal and not only for its behavior over $T^{c}$. Of course, if $x^{*}$ is the solution of $i \mathrm{BPDN}$ (with $\epsilon=0$ ), we get similarly

$$
\left\|x_{T^{c}}-x_{T^{c}}^{*}\right\|_{2} \leq\left\|x-x^{*}\right\|_{2} \leq D_{s, k} e_{0}(r, k)
$$

with $D_{s, k}<2 \frac{1+(\sqrt{2}-1) \delta_{s+2 k}}{1-(\sqrt{2}+1) \delta_{s+2 k}}<\widetilde{D}_{\delta^{\prime}}$.

We can remark also that, conversely to the current cancel-then-recover strategy, $i$ BPDN provides stability against noisy measurements. An open question is however that $\Phi$ in $[9$ has not to be really RIP of order $s+2 k$ to valid (3). As reported in Lemma 1, $\Phi$ simply needs to provide a $\delta$-stable embedding over $\left(\Sigma_{2 k}, \Sigma_{T}\right)$ which is weaker than asking the RIP of order $s+2 k$. Given $k$ and $m$, that second requirement holds possibly for a smaller radius $\delta$ than the RIP radius $\delta_{s+2 k}$.

\section{Numerical Method}

In this section, we sketch of a simple algorithm for the reader interested in a numerical implementation of $i \mathrm{BPDN}$. This one relies on monotone operator splitting and proximal methods [8, 11].

\footnotetext{
${ }^{4}$ Robustness of this strategy against an additional noise $n$ could be obtained by bounding the power of $P_{\Omega^{\perp}} n$ when $y=\Phi x+n$.
} 
At the heart of this procedure is the definition of the proximity operator of any convex function $\varphi: \mathbb{R}^{n} \rightarrow \mathbb{R}$, i.e. the unique solution of $\operatorname{prox}_{\varphi}(z)=\arg \min _{u} \frac{1}{2}\|u-x\|_{2}^{2}+\varphi(z)$.

Both BPDN and $i$ BPDN are special cases of the general minimization problem

$$
\arg \min _{x \in \mathcal{H}} f(x)+g(x) .
$$

For $i \mathrm{BPDN}, f(u)=\left\|u_{T^{c}}\right\|_{1}$ and $g(u)=\imath_{C(\epsilon)}(u)=0$ if $u \in C(\epsilon)$ and $\infty$ otherwise, i.e. the indicator function of the closed convex set $C(\epsilon)=\left\{v \in \mathbb{R}^{n}:\|y-\Phi v\|_{2} \leq \epsilon\right\}$.

Of course $f$ and $g$ are both non-differentiable, however, since (i) their domain is non-empty, (ii) they are convex and (iii) lower semi-continuous (lsc), i.e. $\liminf _{u \rightarrow u_{0}} f(u)=f\left(u_{0}\right)$ for all $u_{0} \in$ $\operatorname{dom} f, i \mathrm{BPDN}$ can be solved by the following Douglas-Rachford iterative method [11]:

$$
u^{(t+1)}=\left(1-\frac{\alpha_{t}}{2}\right) u^{(t)}+\frac{\alpha_{t}}{2} S_{\gamma}^{\odot} \circ \mathcal{P}_{C(\epsilon)}^{\odot}\left(u^{(t)}\right),
$$

where $A^{\odot} \triangleq 2 A-\mathrm{Id}$ for any operator $A, \alpha_{t} \in(0,2)$ for all $t \in \mathbb{N}, S_{\gamma}=\operatorname{prox}_{\gamma f}$ for some $\gamma>0$ and $\mathcal{P}_{C(\epsilon)}=\operatorname{prox}_{g}$ is the orthogonal projection onto the tube $C(\epsilon)$. From [7], one can show that the sequence $\left(u^{(t)}\right)_{t \in \mathbb{N}}$ converges to some point $u^{*}$ and $x^{*}=\mathcal{P}_{C(\epsilon)}\left(u^{*}\right)$ is the solution of $i$ BPDN.

We may compute that $S_{\gamma} z=\operatorname{prox}_{\gamma f} z$ is actually the component-wise soft-thresholding operator of $z$ on $T^{c}$, i.e. $\left(S_{\gamma} z\right)_{i}=\operatorname{sign} z_{i}\left(\left|z_{i}\right|-\gamma\right)_{+}$if $i \in T^{c}$ and $z_{i}$ if $i \in T$, with, for $\lambda \in \mathbb{R},(\lambda)_{+}=\lambda$ if $\lambda \geq 0$ and 0 else. Efficient ways to compute $\mathcal{P}_{C(\epsilon)}$ are also given in [11.

\section{Conclusion}

This short note has studied the modification of Compressed Sensing introduced in [16, 17, i.e. when the signal sparsity assumption is increased by the knowledge of a part of its support. We showed theoretically that a simple generalization of the common Basis Pursuit DeNoise program, i.e. the innovative BPDN, has similar stability guarantees than BPDN with respect to both signal compressibility and noisy measurements. Interestingly, the obtained requirements are related to the conclusion of [9] when the cancel-then-recover strategy is applied to the context of this paper.

In the future, we plan to investigate possible numerical applications of this formalism. In particular, when $i \mathrm{BPDN}$ is integrated to the reconstruction of sequences of sparse or compressible signals, we would like to assess the quality of the reconstruction in function of the number of measurements when the amount of innovation, i.e. the ratio between the unknown and the known signal support parts, can be quantified over time.

\section{Acknowledgements}

We are very grateful to Prof. Pierre Vandergheynst (Signal Processing Laboratory, LTS2/EPFL, Switzerland) for his useful advices and his hospitality during their postdoctoral stay in EPFL.

\section{References}

[1] E.J. Candès. The restricted isometry property and its implications for compressed sensing. Compte Rendus de l'Academie des Sciences, Paris, Serie I, 346:589-592, 2008. 
[2] E.J. Candès and J. Romberg. Quantitative Robust Uncertainty Principles and Optimally Sparse Decompositions. Foundations of Computational Mathematics, 6(2):227-254, 2006.

[3] E.J. Candès, J. Romberg, and T. Tao. Stable signal recovery from incomplete and inaccurate measurements. Comm. Pure Appl. Math, 59(8):1207-1223, 2006.

[4] E.J. Candès and T. Tao. Decoding by linear programming. Information Theory, IEEE Transactions on, 51(12):4203-4215, 2005.

[5] S. Shaobing Chen, D.L. Donoho, and M.A. Saunders. Atomic decomposition by basis pursuit. SIAM Journal on Scientific Computing, 20(1):33-61, 1998.

[6] A. Cohen, R. DeVore, and W. Dahmen. Compressed sensing and best k-term approximation. J. Amer. Math. Soc., 22:211-231, 2009.

[7] P.L. Combettes. Solving monotone inclusions via compositions of nonexpansive averaged operators. Optimization, 53(5):475-504, 2004.

[8] P.L. Combettes and J.C. Pesquet. A Douglas-Rachford Splitting Approach to Nonsmooth Convex Variational Signal Recovery. Selected Topics in Signal Processing, IEEE Journal of, $1(4): 564-574,2007$.

[9] Mark A. Davenport, Petros T. Boufounos, Michael B. Wakin, and Richard G. Baraniuk. Signal processing with compressive measurements. to appear in Journal of Selected Topics in Signal Processing, 2010.

[10] D.L. Donoho. Compressed Sensing. Information Theory, IEEE Transactions on, 52(4):12891306, 2006.

[11] M.J. Fadili and J.-L. Starck. Monotone operator splitting for fast sparse solutions of inverse problems. SIAM Journal on Imaging Sciences, 2009. submitted.

[12] S. Mallat. A Wavelet Tour of Signal Processing. Academic Press., 2nd ed edition, 1999.

[13] B.K. Natarajan. Sparse Approximate Solutions to Linear Systems. SIAM Journal on Computing, 24:227, 1995.

[14] D. Needell and J. A. Tropp. Cosamp: Iterative signal recovery from incomplete and inaccurate samples. Appl. Comp. Harmonic Anal., 26:301-321, 2008. arXiv math.NA 0803.2392.

[15] J. A. Tropp. Just relax: Convex programming methods for identifying sparse signals. IEEE Trans. Info. Theory, 51(3):1030-1051, 2006.

[16] N. Vaswani and W. Lu. Modified-CS: Modifying Compressive Sensing for Problems with Partially Known Support. 2009. Arxiv preprint, available at http://arxiv.org/abs/0903. 5066 .

[17] Namrata Vaswani and Wei Lu. Modified-cs: Modifying compressive sensing for problems with partially known support. In IEEE Intl. Symp. Info. Theory (ISIT), 2009. 